\title{
EchoGéo
}

29 | 2014

The Political Ecology of Conservation and

Development Territories

\section{Les langues de la géographie}

\section{Alexis Sierra}

\section{(2) OpenEdition}

1 Journals

Electronic version

URL: https://journals.openedition.org/echogeo/13947

DOI: $10.4000 /$ echogeo.13947

ISSN: 1963-1197

Publisher

Pôle de recherche pour l'organisation et la diffusion de l'information géographique (CNRS UMR 8586)

\section{Electronic reference}

Alexis Sierra, "Les langues de la géographie", EchoGéo [Online], 29 | 2014, Online since 03 October 2014, connection on 31 July 2021. URL: http://journals.openedition.org/echogeo/13947 ; DOI: https:// doi.org/10.4000/echogeo.13947

This text was automatically generated on 31 July 2021.

EchoGéo est mis à disposition selon les termes de la licence Creative Commons Attribution - Pas d'Utilisation Commerciale - Pas de Modification 4.0 International (CC BY-NC-ND) 


\title{
Les langues de la géographie
}

\author{
Alexis Sierra
}

1 La géographie, science sociale, relève également de la littérature. Différentes langues assurent sa diffusion et proposent des mots qui sont autant d'occasions pour conceptualiser cette science. L'utilisation de telle ou telle langue pour analyser, problématiser, diffuser n'est pas anodine. En France, bien des «mots de la géographie » pour reprendre le titre d'un dictionnaire français qui a fait date ${ }^{1}$, sont issus de langues étrangères. Ils montrent les circuits de construction du savoir-penser l'espace et de sa diffusion, de l'allemand inselberg ou hinterland à l'anglais edge city en passant par l'espagnol ria ou altiplano, et l'arabe oued. La fortune de tel ou tel terme allophone témoigne de conceptualisations opérées à partir de terrains spécifiques ou d'une recherche menée en coopération avec des partenaires étrangers. En ce sens, la géographie, discipline tournée vers l'ailleurs, contribue à l'enrichissement et à la diversification d'un vocabulaire scientifique dont les origines sont multiples. Les études urbaines, pluridisciplinaires, témoignent notamment de cette "aventure des mots", « invitation à de multiples cheminements possibles dans les villes et dans les mots, dans le temps, les langues, les sociétés urbaines» et ce pour essayer de restituer la signification que donnent les populations à des mots du quotidien².

2 L'intérêt pour l'épistémologie a également conduit à s'intéresser aux évolutions de la discipline à l'étranger et à importer à partir de la crise de la fin des années soixante des termes anglais pour réfléchir au renouveau de la géographie. C'est ainsi que la réutilisation de certaines notions souligne elle-même la filiation anglo-saxonne de courants développés en France en faisant référence aussi bien à la New geography qu'à la humanistic geography. La démarche réflexive, le postmodernisme, le courant postcolonial, le désir de décloisonner la discipline, de la refonder, sont autant de facteurs qui ont conduit les géographes français à s'intéresser à la littérature anglo-saxonne, littérature qui a d'ailleurs elle-même des origines variées et ne se réduit pas, même si elle est majoritaire, à celle venue des États-Unis. Un autre dictionnaire de géographie française de référence, en donnant systématiquement la traduction des notions en anglais et en allemand, témoigne en partie de cette volonté de décloisonnement tout en privilégiant deux langues et donc deux foyers de savoirs épistémologiques ${ }^{3}$. 
Cependant, les enjeux de l'usage de telle ou telle langue sont plus profonds, liés d'une part à l'injonction de lire et publier en anglais et d'autre part, à la nécessité d'échanger facilement au sein d'une communauté scientifique internationale dont l'anglais est devenu la langue véhiculaire.

4 C'est donc très justement qu'Antoine Le Blanc et Nathalie Lemarchand, vice-présidents au Comité national Français de géographie et en charge des manifestations et des relations internationales, ont ouvert un riche débat sur la place du français dans la production et la diffusion du savoir scientifique, notamment en relation avec la langue dominante aujourd'hui qu'est l'anglais. Dans la synthèse qui suit, ils nous livrent l'intérêt et l'actualité d'un tel débat, rappelant les thèmes abordés lors de la rencontre internationale qu'ils ont organisé en juillet dernier. Ils y font état de nombreuses problématiques soulevées à cette occasion et nous invitent à les approfondir et les enrichir dans les mois qui viennent. Des réseaux tels que le CNFG et au-delà l'Union Internationale des géographes (UGI) sont et seront nécessairement confrontés à cette question de la langue dans laquelle non seulement des savoirs sont diffusés mais également dans laquelle ils sont élaborés.

\section{NOTES}

1. Brunet R., 1992. Les mots de la géographie, dictionnaire critique. Montpellier-Paris, Reclus-La Documentation française.

2. Topalov C. et al., 2010. L'aventure des mots de la ville, à travers le temps, les langues, les sociétés. Paris, Robert Laffont.

3. Lévy J., Lussault M., 2003. Dictionnaire de géographie et de l'espace des sociétés. Paris, Belin.

\section{AUTHOR}

\section{ALEXIS SIERRA}

Alexis Sierra, alexisierra2001@yahoo.fr, est Maître de conférences en géographie à l'université

Cergy-Pontoise-IUFM. 